\title{
3D Nondestructive Visualization and Evaluation of TRISO Particles Distribution in HTGR Fuel Pebbles Using Cone-Beam Computed Tomography
}

\author{
Gongyi Yu, Yi Du, Xincheng Xiang, Yuan Liu, Ziqiang Li, and Xiangang Wang \\ Institution of Nuclear and New Energy Technology, Tsinghua University, Beijing 100084, China \\ Correspondence should be addressed to Xiangang Wang; wangxiangang@tsinghua.edu.cn
}

Received 27 December 2016; Accepted 26 February 2017; Published 7 March 2017

Academic Editor: Robert Morris

Copyright (C) 2017 Gongyi Yu et al. This is an open access article distributed under the Creative Commons Attribution License, which permits unrestricted use, distribution, and reproduction in any medium, provided the original work is properly cited.

\begin{abstract}
A nonuniform distribution of tristructural isotropic (TRISO) particles within a high-temperature gas-cooled reactor (HTGR) pebble may lead to excessive thermal gradients and nonuniform thermal expansion during operation. If the particles are closely clustered, local hotspots may form, leading to excessive stresses on particle layers and an increased probability of particle failure. Although X-ray digital radiography (DR) is currently used to evaluate the TRISO distributions in pebbles, X-ray DR projection images are two-dimensional in nature, which would potentially miss some details for 3D evaluation. This paper proposes a method of $3 \mathrm{D}$ visualization and evaluation of the TRISO distribution in HTGR pebbles using cone-beam computed tomography (CBCT): first, a pebble is scanned on our high-resolution CBCT, and 2D cross-sectional images are reconstructed; secondly, all crosssectional images are restructured to form the 3D model of the pebble; then, volume rendering is applied to segment and display the TRISO particles in 3D for visualization and distribution evaluation. For method validation, several pebbles were scanned and the 3D distributions of the TRISO particles within the pebbles were produced. Experiment results show that the proposed method provides more 3D than $\mathrm{DR}$, which will facilitate pebble fabrication research and production quality control.
\end{abstract}

\section{Introduction}

High-temperature gas-cooled reactor (HTGR) is one of the six candidates of the Generation IV nuclear systems which attract many countries' interests. One of the important safety features of the HTGR is the robust fission product retention capabilities of the fuel elements [1]. One of the important features of the HTGR is the improved safety of fuel elements. The Chinese High-Temperature Reactor Pebble-bed Module (HTR-PM) uses pebbles as fuel elements, which may enclose more than ten thousand tristructural isotropic (TRISO) particles in a single pebble [2]. The TRISO particle is the first and most important barrier to retain fission products $[1,2]$. The distribution of TRISO particles within the pebbles is an important factor for the safety and fuel performance evaluation of the pebbles, because nonuniform distribution of particles causes nonuniform distribution of temperature gradient and produces thermal expansions within a pebble. It is therefore important to evaluate the particle distributions within a pebble to be certain that the nonuniformity of the distributions does not exceed certain criteria.

Fabrication of pebbles is a very complicated process [2-4]. Firstly, TRISO particles and graphite powder are completely mixed and prepressed to form a ball with a silicone rubber mold. Secondly, a graphite ball with TRISO particles is formed in the fuel zone by cold quasi-isostatic pressing. Thirdly, the $5 \mathrm{~mm}$ graphite layer is pressed outside the fuel zone to form the nonfuel zone to provide protection for the TRISO particles. And finally, after the process of lathing, the heat treatments of carbonization, and high-temperature purification, the fuel pebble is formed. Figure 1 illustrates the structure of a pebble. A large number of TRISO particles are distributed randomly in a graphite matrix in the inner part of the pebble, which is about $50 \mathrm{~mm}$ in diameter. The outer part of the pebble is a layer of graphite that does not contain any fuel.

To visualize the particle distribution, X-ray Nondestructive Testing (NDT), such as the X-ray digital radiography 


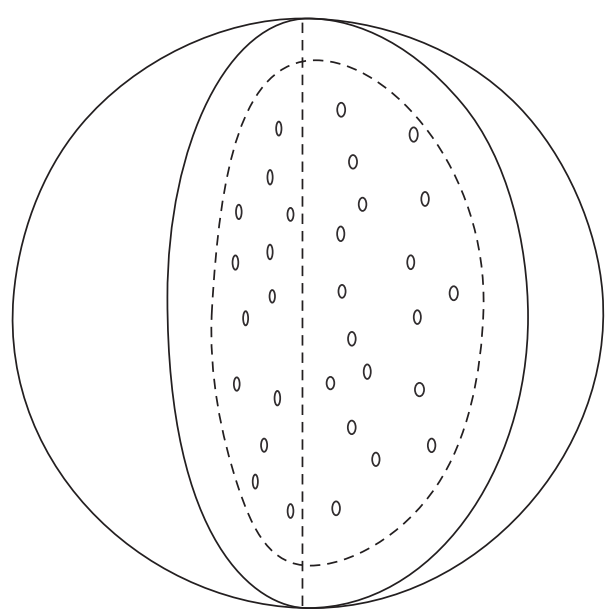

FIGURE 1: Scheme of a pebble.

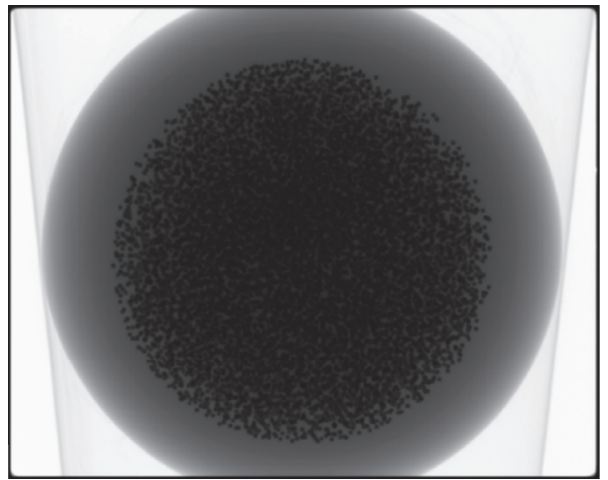

FIgURE 2: Raw DR of a fuel pebble.

(DR), is conventionally used at INET, Tsinghua University, because it is fast and penetrating. Figure 2 shows a digital radiography of a fuel pebble with the particles indicated by the black dots. These features make it convenient to evaluate parameters such as the size of the nonfuel zone [4-6].

Although DR can provide a preliminary evaluation of the particle distribution, DR is only a $2 \mathrm{D}$ projection of the $3 \mathrm{D}$ distribution of the TRISO particles. Particles overlap each other, which leads to a confusing image in a $2 \mathrm{D}$ projection. Moreover, the cracks or other structures with small effective cross section parallel to the direction of X-ray cause even more confusion in the DR image. For the DR method, welldistributed TRISO particles in the fuel zone cannot produce clear images. For example, we could not find the cutting edge of TRISO in Figure 3. In the $2 \mathrm{D}$ image produced by the DR method, the intensities of the particles depend on the thickness that X-ray penetrates instead of the number of particles in a certain volume.

The TRISO particles are desired to be uniformly distributed within the pebble so that they do not touch each other. The TRISO particles, however, are often distributed nonuniformly because they are not fully mixed when packing together into a pebble during the fabrication process.
Destructive methods such as the sample-slice-polish method are a conventionally used option to assess particle distributions. The sample is sliced and the section polished to take a photographic picture of the cross section in $2 \mathrm{D}$. This method is straightforward to implement and the image can be in high resolution. It is, however, time-consuming, labor-intensive, operator-dependent, and not intrinsically a 3D method $[7,8]$. More importantly, this method requires the pebbles to be cut into slices and causes difficulties in dust control. An alternative nondestructive method is therefore desired. The conventionally used DR method, however, cannot reliably assess the particle distribution due to the aforementioned disadvantages [7]. It is therefore important to develop a new nondestructive method for this purpose.

In this work, we used $3 \mathrm{D}$ cone-beam CT to acquire $3 \mathrm{D}$ tomographic images of particle distributions within pebbles. This method provides more valuable information to assess pebble quality [9].

\section{Method}

Cone-beam CT reconstructs $3 \mathrm{D}$ slices from $360^{\circ}$ projections. The scheme of $3 \mathrm{D}$ cone-beam CT is shown in Figure 4, which 


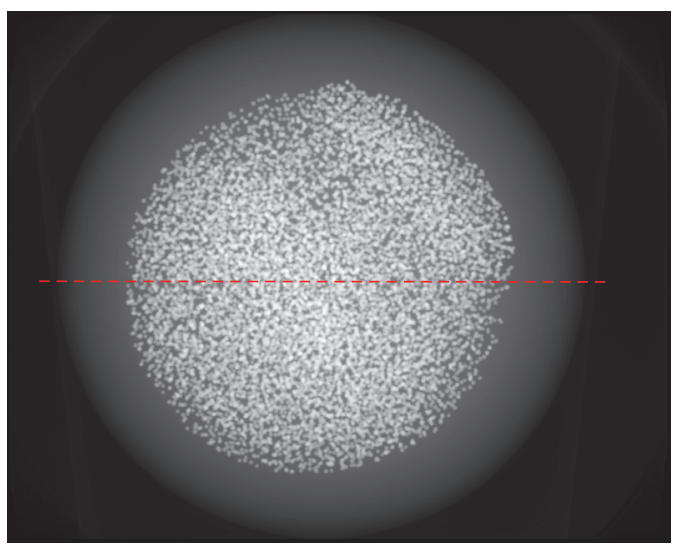

(a)

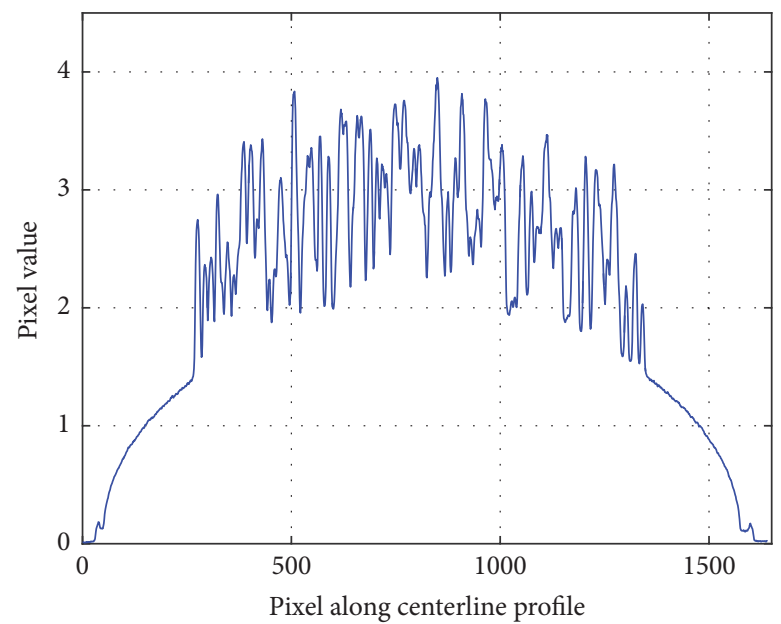

(b)

FIgURE 3: (a) DR of a fuel pebble and (b) its centerline profile marked in (a).

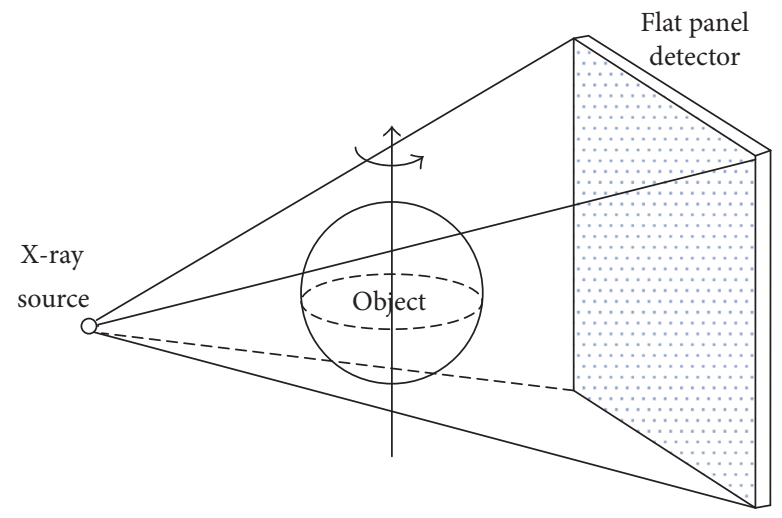

(a)

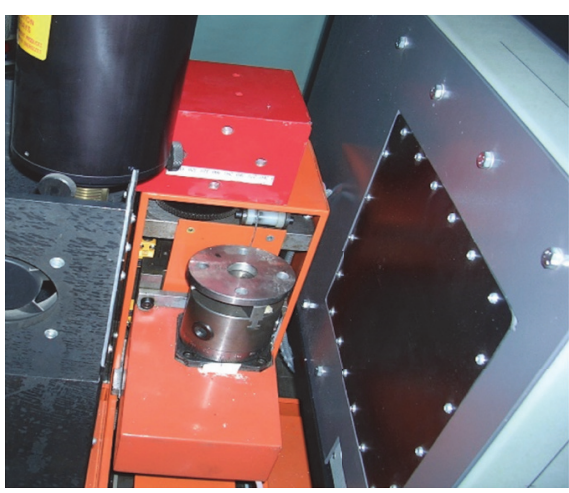

(b)

FIGURE 4: Scheme of flat panel based 3D cone-beam CT system and facility.

has good spatial resolution, efficient scanning, and effective $\mathrm{X}$-ray utilization [9]. The system uses a $160 \mathrm{kVp}$ microfocus $\mathrm{X}$-ray generator and a flat panel detector. The X-ray tube is HOMX-161 microfocus tube by Philips. The voltage and current of the X-ray tube for pebble imaging are $140 \mathrm{kV}$ and $0.2 \mathrm{~mA}$, respectively. The focus spot is about 20 50 $\mu \mathrm{m}$. The flat panel detector is PaxScan 2520 by Varian. The detector has $1920 \times 1536$ pixels with a pitch of $127 \mu \mathrm{m} \times 127 \mu \mathrm{m}$. The distance between the source and the detector is about $75 \mathrm{~cm}$ and the source-object distance is $25 \mathrm{~cm}$. The equivalent pixel size of the slice is about $80 \mu \mathrm{m}[9,10]$.

A series of projections are acquired while scanning, and the time varies from 10 to 30 minutes per pebble. Slices are reconstructed with the $3 \mathrm{D}$ CBCT reconstruction algorithm, and the time may vary from 5 to 10 minutes if accelerated by GPUs. The reconstruction algorithm we used is fast FDK [11], which is in-house programmed in Visual Studio 2012. The flow chart for the visualization process is shown in Figure 5. A fuel pebble is scanned with $\mathrm{CT}$, and the reconstruction with acquired projections is simultaneously performed while scanning.

As a key step for 3D evaluation, the TRISO particle distribution is displayed in a $3 \mathrm{D}$ view by using visualization rendering. With visualization rendering, a complex $3 \mathrm{D}$ structure can be demonstrated in a more intuitive way. Surface rendering and volume rendering are two typical visualization methods. Surface rendering extracts polygonal mesh of an iso-surface from three-dimensional voxels. Volume rendering, such as ray-casting, works on the basis of pixel and it presents more details.

With TRISO particles displayed in a 3D and intuitive way, it becomes much easier for fuel experts to judge the TRISO distribution as good or bad, accepted or rejected. In addition, the CT modality and NDT staff establish qualified slices.

In this work, two HTR-PM pebbles were scanned to demonstrate feasibility using the 3D cone-beam CT with an angle step of 0.5 degrees, and hence 720 projections were made for each pebble. The scanning took about 20 


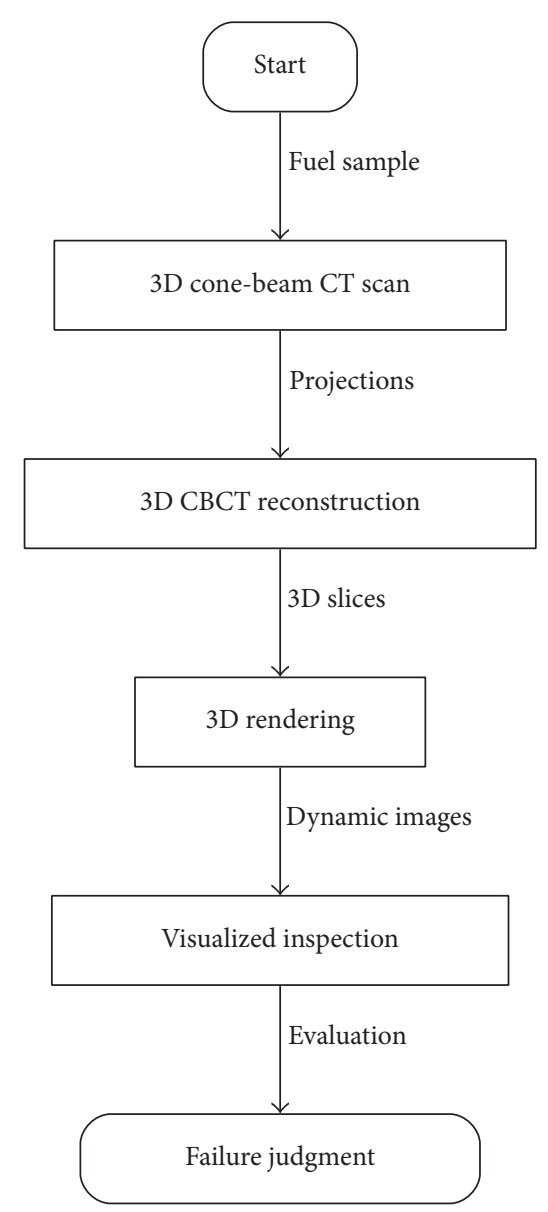

FIGURE 5: 3D visualized inspection process.

minutes, and the reconstruction was done simultaneously. $3 \mathrm{D}$ rendering and animation were processed using the raycasting module in Amira (version 5.4).

\section{Experimental Result and 3D Visualization Evaluation}

Slices were reconstructed with the FDK algorithm and the central slice is shown in Figure 6. The distribution of TRISO particle is shown in 2D. Three slices in $X, Y$, and $Z$ directions are shown, respectively.

Figure 7 is a $3 \mathrm{D}$ volume rendering of TRISO in a pebble. The particles can be individually identified, and the space distribution is clear.

\section{Discussion}

To conveniently inspect the uniformity of TRISO distribution, several attributes, such as color, transparency, illumination, and perspective, could be set in the rendered images as shown in Figure 8.

Animation is also applicable as shown in Figure 9 and in the Supplementary Material (available online at https://doi.org/10.1155/2017/3857075). With expert evaluation, aggregation phenomena could be observed easily. By using this tool, experts can identify any suspected defect regions from any view and the associated possible causes due to the fabrication process. The results provided by this method can therefore help optimize the manufacture process [12]. The results also provide information about the initial conditions of the pebbles for the fuel performance evaluations.

\section{Conclusions}

As the first layer of HTGR intrinsic safety system, the fuel pebble is fabricated in a complicated processing chain. For production QA (quality assurance) and QC (quality control), each process parameter of fuel element should be carefully defined and evaluated. The images generated by X-ray DR are two-dimensional and not well qualified to evaluate the $3 \mathrm{D}$ TRISO particle distribution.

This study provides a viable CBCT-based nondestructive method to visualize and evaluate the TRISO distribution in three dimensions. Experiment results showed that $3 \mathrm{D}$ conebeam CT provides detailed 3D information compared to DR, which helps experts to individually evaluate each fuel 


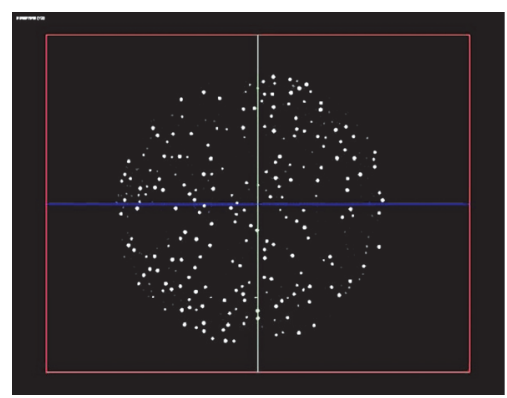

(a) $X-Y$ slice

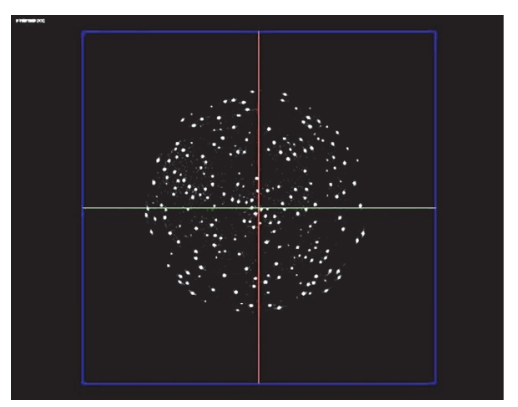

(b) $X-Z$ slice

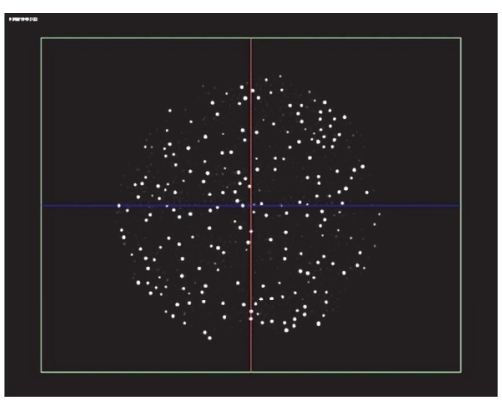

(c) $Y$-Z slice

FIGURE 6: Distribution of TRISO particles in 2D.

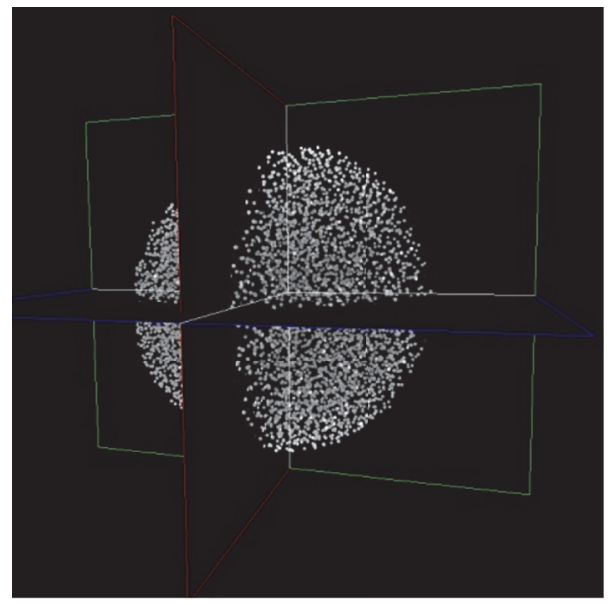

FIGURE 7: 3D visualization of TRISO particles in a pebble.

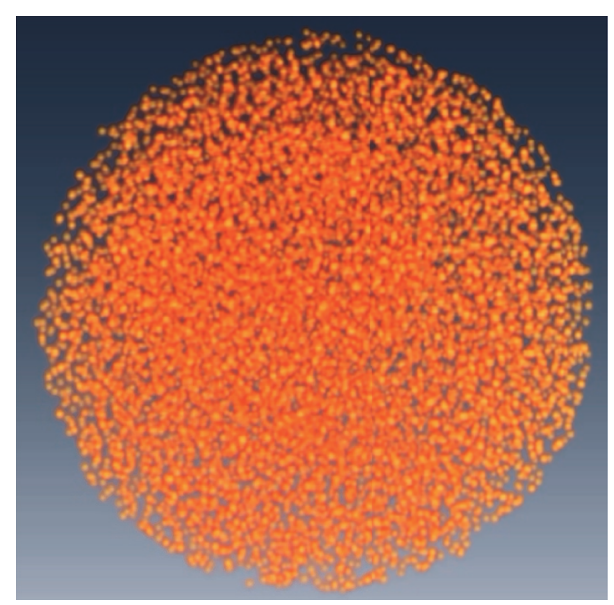

FIgURE 8: Adjustable 3D visualization of particles in a pebble.

element slice. Experts can also visualize 3D data animatedly by the volume rendering technique, which helps the expert to evaluate the TRISO distribution or fabrication defects (e.g., cracks) in a more direct way. It also helps in aggregation or some other complicated circumstances, such as process optimization and quality control.

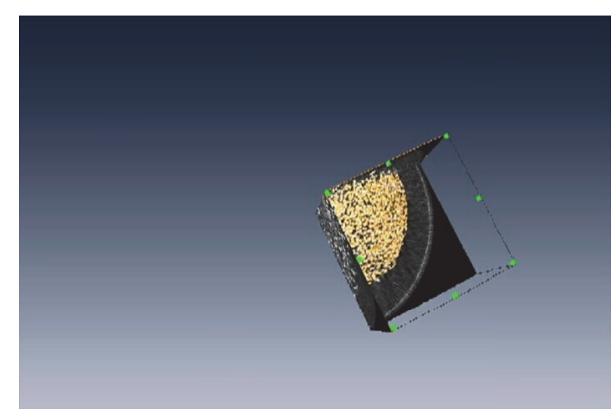

FIGURE 9: Illustration of animated 3D visualization of 3D slices of fuel element.

Work is in progress to provide statistical evaluations for the particle distributions within a pebble and modeling based on the 3D slices, and the results will be reported separately.

\section{Conflicts of Interest}

The authors declare that there are no conflicts of interest regarding the publication of this paper. 


\section{Acknowledgments}

This paper is partially supported by the National Natural Science Foundation of China (61171115, 61571262, and 11575095) and the National Key Research and Development Program (2016YFC0105406).

\section{References}

[1] International Atomic Energy Agency and Nuclear Power Technology Development Section, "Advances in high temperature gas cooled reactor fuel technology," IAEA-TECDOC 1674, International Atomic Energy Agency (IAEA), 2012.

[2] Z. Zhang, Z. Wu, D. Wang et al., "Current status and technical description of Chinese $2 \times 250 \mathrm{MW}_{\text {th }}$ HTR-PM demonstration plant," Nuclear Engineering and Design, vol. 239, no. 7, pp. 1212$1219,2009$.

[3] Z. Xiangwen, L. Zhenming, Z. Jie et al., "Preparation of spherical fuel elements for HTR-PM in INET," Nuclear Engineering and Design, vol. 263, pp. 456-461, 2013.

[4] K. Verfondern, H. Nabielek, and J. M. Kendall, "Coated particle fuel for high temperature gas cooled reactors," Nuclear Engineering and Technology, vol. 39, no. 5, pp. 603-616, 2007.

[5] M. Yang, R. Li, J. Duan et al., "Analysis of DR testing blind zone of spherical fuel elements for $10 \mathrm{MW}$ high-temperature gascooled reactor," NDT \& E International, vol. 60, pp. 77-86, 2013.

[6] J. Li and Z. Zhang, "X-ray real-time image detection of the fuelfree zone of spherical fuel elements," Physics Examination and Testing, vol. 3, pp. 41-43, 2000.

[7] D. Yi, X. Wang, X. Xiang, and L. Bing, "Automatic X-ray inspection for the HTR-PM spherical fuel elements," Nuclear Engineering and Design, vol. 280, pp. 144-149, 2014.

[8] D. Tisseur, J. Banchet, P.-G. Duny, M.-P. Vitali, G. Peix, and J.-M. Létang, "Quality control of High Temperature Reactors (HTR) compacts via X-ray tomography," in Proceedings of the International Congress on Advances in Nuclear Power Plants. The Nuclear Renaissance at Work (ICAPP '07), pp. 2353-2359, Nice Acropolis, France, May 2007.

[9] L. Zhang, "Determine the homogeneity of UO2 distribution in HTGR fuel element by X-ray tomography," Nuclear Power Engineering, vol. 13, no. 2, pp. 63-68, 1992.

[10] P. R. Hania, B. Janssen, A. V. Fedorov et al., "Qualification of HTR pebbles by X-ray tomography and thermal analysis," Nuclear Engineering and Design, vol. 251, pp. 47-52, 2012.

[11] X. Wang, Z. Guo, Y. Peng, C. Zhang, and J. Tian, "Fast 3$\mathrm{D}$ reconstruction of CT images using a geometric parameter table," Journal of Tsinghua University, vol. 42, no. 5, pp. 655-658, 2002.

[12] International Atomic Energy Agency, "High temperature gas cooled reactor fuels and materials," Tech. Rep. IAEA-TECDOCCD-1645, International Atomic Energy Agency (IAEA), Vienna, Austria, 2010. 

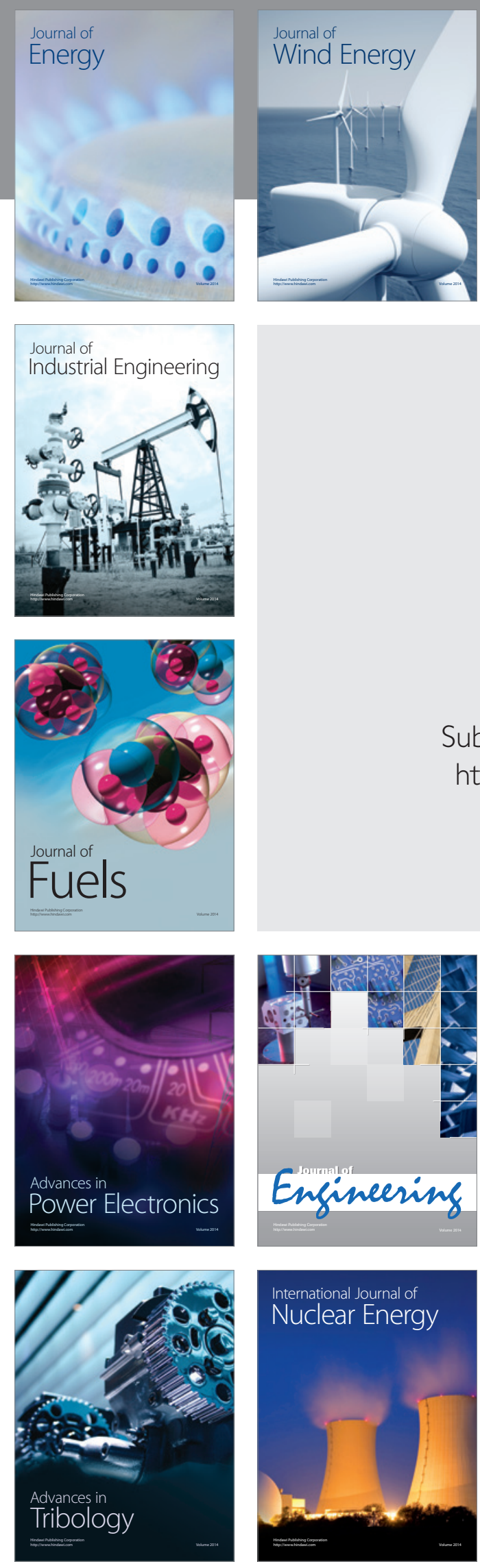

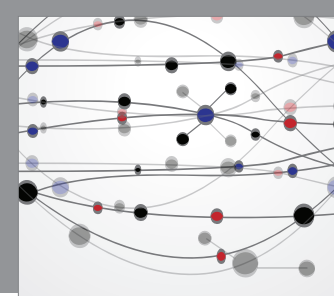

The Scientific World Journal
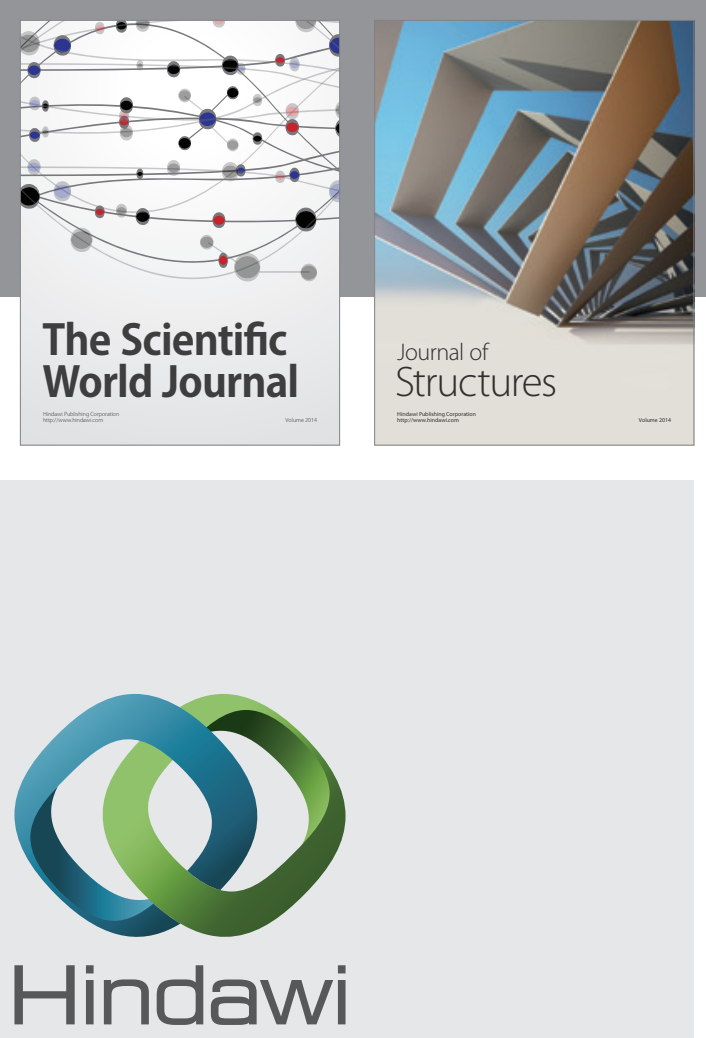

Submit your manuscripts at

https://www.hindawi.com
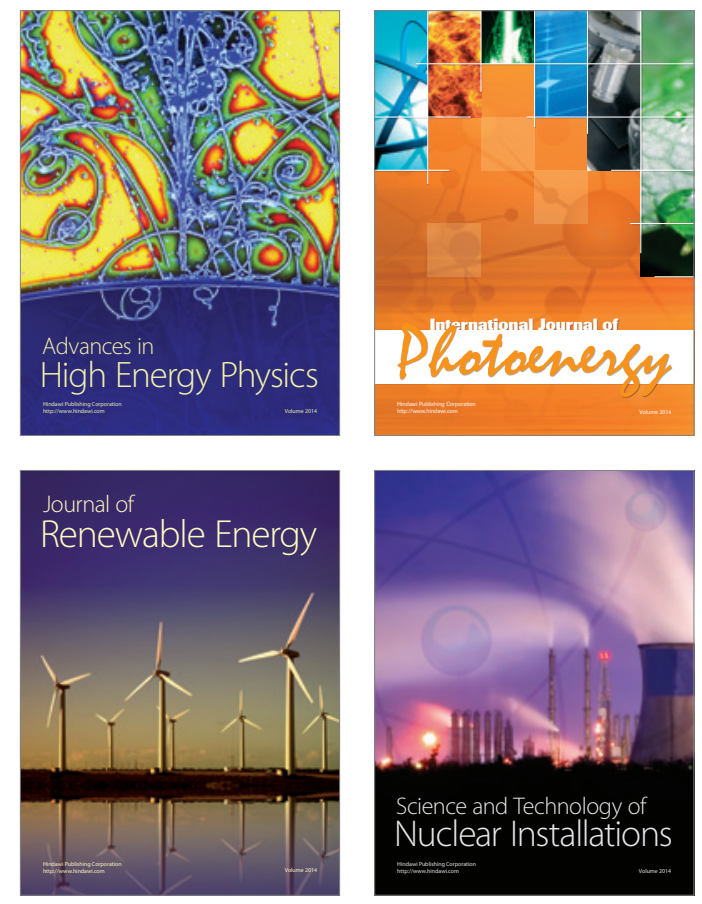
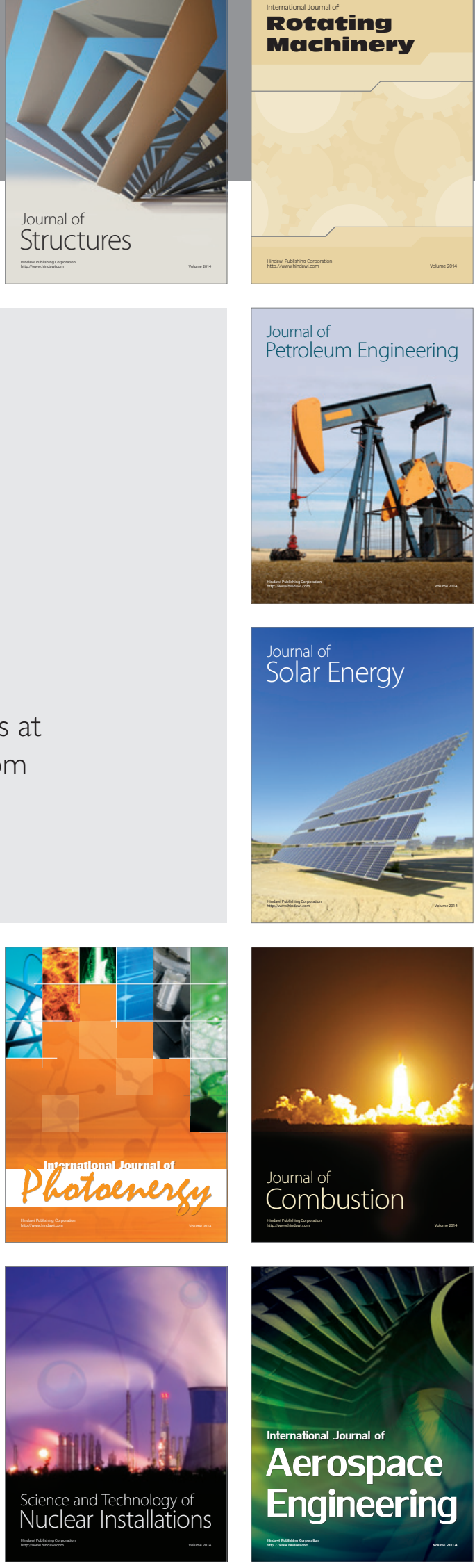